\title{
Chaotic Convective Behavior and Stability Analysis of a Fractional Viscoelastic Fluids Model in Porous Media
}

\author{
Ibrahima N'Doye and Taous-Meriem Laleg-Kirati
}

\begin{abstract}
In this paper, a dynamical fractional viscoelastic fluids convection model in porous media is proposed and its chaotic behavior is studied. A preformed equilibrium points analysis indicates the conditions where chaotic dynamics can be observed, and show the existence of chaos. The behavior and stability analysis of the integer-order and the fractional commensurate and non-commensurate orders of a fractional viscoelastic fluids system, which exhibits chaos, are presented as well.
\end{abstract}

Index Terms-Fractional calculus, generalized fractionalorder system, thermal convection, viscoelastic fluids, chaotic convective behavior, porous media.

\section{INTRODUCTION}

Modeling the thermal convection has attracted the interest of engineers and scientists for a long time due to its numerous applications [1]. Understanding of the thermal convective behavior is important for controlling processes like the utilization of geothermal energy such as building thermal insulation. Several modeling approaches have been proposed including the viscoelastic model which seems to fit well the experimental data in some situations [2]. Chaotic behavior can be found in thermal convection. The analysis of this behavior started by the work of Lorenz [3] followed by some experimental validation [4] and several other studies (see [5], [6], [7], [8], [9], [10], [11] and references therein).

Fractional calculus has some interesting properties such as long memory and hereditary properties, which make it an efficient modeling tool in porous media. It also offers additional degrees of freedom which help in reproducing realistic behaviors. The fractional differentiation order has a direct effect on the chaotic behavior of nonlinear dynamical systems and on the control and performance of fractionalorder systems. Such systems have been considered recently in modeling many phenomena such as viscoelasticity [12], [13], [14], [15]. Recently, the generalized non-Newtonian fluids equations have been modified from the well-known fluid models by replacing the integer-order derivative with the fractional differentiation orders [16], [17]. Chaotic behavior has been studied in fractional systems for instance in [18], [19]. Hartley et al. introduced the fractional-order Chua's system and showed that with the order less than 3 , the chaotic behavior is still possible [20]. In [21], the fractional-order cellular neural network was considered, and

Ibrahima N'Doye and Taous-Meriem Laleg-Kirati are with Computer, Electrical and Mathematical Sciences and Engineering Division (CEMSE), King Abdullah University of Science and Technology (KAUST). Thuwal 23955-6900, KSA. ibrahima.ndoyedkaust.edu.sa, taousmeriem.laleg@kaust.edu.sa the fractional Duffing's system was presented in [22]. The other fractional-order chaotic systems were described in many other references (e.g. [23], [24], [25], etc.).

In this paper we are interested in a fractional viscoelastic fluids where we propose to study the chaotic behavior of the model. We show that the fractional-order model is a good candidate to describe the viscoelastic fluid in porous media. We show also that this model can be reduced to some systems such as the fractional-order Khayat's system, the fractionalorder Lorenz's system [26], the fractional-order Vadasz's system, and the fractional-order Akhatov's system. We also study the effect of the fractional differentiation order on the chaotic behavior and compare the results to the integer-order model.

This paper is organized as follows : In section II, the basic definition and some preliminaries on fractional calculus are given. Section III introduces the integer and fractional-order models of the thermal convection in a viscoelastic fluidsaturated porous medium. Then the fractional-order model is studied by using the stability theory of fractional-order systems, the bifurcation analysis and numerical simulations. The effect of the fractional differentiation commensurate and non-commensurate orders on the chaotic behavior is studied. The effect of Darcy-Rayleigh number on the fractional model dynamics and on the transition from steady convection to chaos is also illustrated. Concluding remarks are given in section IV.

\section{PRELIMINARIES}

\section{A. Definition of the fractional derivatives}

There are several definitions of fractional derivative in the literature. The most used ones are the Caputo, the RiemannLiouville and the Grünwald-Letnikov [27]. In this paper we use the Grünwald-Letnikov derivative. The latter is defined by the fractional differentiation order $\alpha$ of function $f(t)$ with respect to $t$ and the terminal value 0 :

${ }_{0} D_{t}^{\alpha} f(t)=\frac{\mathrm{d}^{\alpha} f(t)}{\mathrm{d} t^{\alpha}}=\lim _{t \rightarrow 0} h^{-\alpha} \sum_{j=0}^{\left[\frac{t}{n}\right]}(-1)^{j}\left(\begin{array}{l}\alpha \\ j\end{array}\right) f(t-j h)$,

with $n \in \mathbb{N}$ and $\alpha \in \mathbb{R}^{+}$, [.] means the integer part.

In the rest of the paper, we use an operator ${ }_{0} D_{t}^{\alpha}$ to represent the Grünwald-Letnikov derivative with zero initial value history.

\section{B. Numerical simulations of fractional differential equations}

For numerical simulation of the fractional-order systems, we propose to use the Grünwald-Letnikov method [23], 
[28] based on the Adams-Bashforth-Moulton type predictorcorrector scheme [29]. The relation for the explicit numerical approximation of the $\alpha^{t h}$ derivative at the points $k h$ (see also [23], [28], [30]) is given by

$$
\left(k-L_{m} / h\right) D_{k h}^{\alpha} f(t) \approx h^{-\alpha} \sum_{j=0}^{k}(-1)^{j}\left(\begin{array}{l}
\alpha \\
j
\end{array}\right) f\left(t_{k}-j\right),
$$

where $L_{m}$ is the memory length, $t_{k}=k h, h$ is the time step of the calculation and $(-1)^{j}\left(\begin{array}{l}\alpha \\ j\end{array}\right)$ are binomial coefficients $c_{j}^{(\alpha)}(j=0,1, \cdots)$ which can be computed as

$$
c_{0}^{(\alpha)}=1, \quad c_{j}^{(\alpha)}=\left(1-\frac{1+\alpha}{j}\right) c_{j-1}^{(\alpha)} .
$$

Numerical solution of the fractional differential equation

$$
{ }_{0} D_{t}^{\alpha} y(t)=f(y(t), t)
$$

can be expressed as [28]

$$
y\left(t_{k}\right)=f\left(y\left(t_{k}\right), t_{k}\right) h^{\alpha}-\sum_{j=1}^{k} c_{j}^{(\alpha)} f\left(t_{k}-j\right) .
$$

Equation (5) is nonlinear with respect to finding $y\left(t_{k}\right)$ and can be solved using any suitable method for such equations.

\section{Fractional-order systems}

Let us consider the following fractional order nonlinear system with zero initial value history in the form [28]

$$
\left\{\begin{array}{c}
{ }_{0} D_{t}^{\alpha_{i}} x_{i}(t)=f_{i}\left(x_{1}(t), x_{2}(t), \cdots, x_{n}(t), t\right) \\
x_{i}(t)=c_{i}, \quad \text { for } \quad i=1,2, \cdots, n
\end{array}\right.
$$

where $c_{i}$ are initial conditions, $\alpha=\left[\alpha_{1}, \alpha_{2}, \cdots, \alpha_{n}\right]^{T}$ are the fractional orders for $0<\alpha_{i}<2,(i=1,2, \cdots, n)$.

An interesting situation is when $\alpha_{1}=\alpha_{2}=\cdots=\alpha_{n}$ and in this case system (6) is called a commensurate order system, otherwise it is a non-commensurate order system.

The vector representation of system (6) can be written as

$$
\mathbf{D}^{\alpha} \mathbf{x}=\mathbf{f}(\mathbf{x})
$$

where $\mathbf{x} \in \mathbb{R}^{n}$. The equilibrium points of system (6) are calculated by solving the following equation

$$
\mathbf{f}(\mathbf{x})=0,
$$

and we denote the equilibrium $x^{*}=\left(x_{1}^{*}, x_{2}^{*}, \cdots, x_{n}^{*}\right)$.

\section{Stability of fractional-order systems}

In the case of nonlinear fractional-order systems, the equilibrium points are asymptotically stable for $\alpha_{1}=\alpha_{2}=\cdots=$ $\alpha_{n} \equiv \alpha$ if all the eigenvalues $\lambda_{i}(i=1,2, \cdots, n)$ of the Jacobian matrix $\mathbf{J}=\partial \mathbf{f} / \partial \mathbf{x}$, where $f=\left[f_{1}, f_{2}, \cdots, f_{n}\right]^{T}$ evaluated at the equilibrium, satisfy the condition [31], [32], [33]

$$
|\arg (\operatorname{eig}(\mathbf{J}))|=\left|\arg \left(\lambda_{i}\right)\right|>\alpha \frac{\pi}{2}, \quad i=1,2 \cdots, n .
$$

For non-commensurate order systems, we use the least common multiple (LCM) of the denominators $u_{i}^{\prime} s$ of $\alpha_{i}^{\prime} s$ denoted $m$ such that $\alpha_{i}=v_{i} / u_{i}, v_{i}, u_{i} \in \mathbb{Z}^{+}$for $(i=1,2, \cdots, n)$ and we set $\gamma=1 / m$, system (7) is asymptotically stable if

$$
|\arg (\lambda)|>\gamma \frac{\pi}{2}
$$

for all roots $\lambda$ of the following equation

$$
\operatorname{det}\left(\operatorname{diag}\left(\left[\begin{array}{ll}
\lambda^{m \alpha_{1}} & \lambda^{m \alpha_{2}} \cdots \lambda^{m \alpha_{n}}
\end{array}\right]\right)-\mathbf{J}\right)=0 .
$$

Fractional-order systems (7) exhibits chaotic behavior if at least one eigenvalue $\lambda$ is in the unstable region [31]. The number of saddle points and eigenvalues for one-scroll, double-scroll and multi-scroll attractors was exactly studied in [32]. Suppose that the unstable eigenvalues of scroll saddle points are: $\lambda=\rho \pm j \beta$. The necessary condition to exhibit one scroll attractor of system (7) is for the eigenvalues $\lambda$ to remain in the unstable region [32]. The condition for commensurate derivative orders is

$$
\alpha>\frac{2}{\pi} \tan ^{-1}\left(\frac{|\beta|}{\rho}\right) .
$$

In other words, when the instability measure $\frac{\pi}{2 m}-$ $\min (|\arg (\lambda)|)$ is negative, the system cannot be chaotic.

\section{CONCEPT OF CHAOTIC CONVECTIVE BEHAVIOR IN A VISCOELASTIC FLUID-SATURATED POROUS MEDIUM}

\section{A. Integer-order system of the thermal convection in a vis- coelastic fluid-saturated porous medium}

Let us consider a thermal convection in a viscoelastic fluidsaturated porous medium [1]. A model has been proposed in [1] resulting from the truncated Galerkin expansion to the momentum and heat transfer equations.

$$
\left\{\begin{array}{l}
\dot{x}_{1}=x_{4} \\
\dot{x}_{2}=R x_{1}-x_{2}-(R-1) x_{1} x_{3} \\
\dot{x}_{3}=4 \gamma\left(x_{1} x_{2}-x_{3}\right), \\
\dot{x}_{4}=\sigma\left[\left(R-\xi^{-1}\right) x_{1}+\left(\xi^{-1}-1\right) x_{2}\right. \\
\left.\quad-(R-1) x_{1} x_{3}-\left(\delta+\frac{1}{\sigma \xi}\right) x_{4}\right]
\end{array}\right.
$$

where $R$ is a modified Darcy-Rayleigh number, $\xi$ is a modified relaxation time, $\sigma$ is a modified Darcy-Prandtl number, $\delta$ is the ratio of retardation to relaxation times and the value of $\gamma$ has to be consistent with the wave number at the convection threshold, which is required so that the convection cells fit into the domain and fulfill the boundary conditions [1].

The system (13) has three equilibrium points :

- $E_{1}=(0 ; 0 ; 0 ; 0)$ which is a trivial solution corresponding to the origin in phase space.

- $E_{2}=(1 ; 1 ; 1 ; 0)$ and $E_{3}=(-1 ;-1 ; 1 ; 0)$ which correspond to the onset of convective roll in opposite directions, as $R$ exceeds unity.

The Jacobian matrix of system (13), evaluated at the equilibrium $E^{*}=\left(x_{1}^{*}, x_{2}^{*}, x_{3}^{*}, x_{4}^{*}\right)$ is given by (14).

Let us consider the following parameters of the system (17) :

$$
R=24, \gamma=0.5, \sigma=100, \xi=1 \text { and } \delta=0.2 .
$$

The corresponding eigenvalues of the Jacobian matrix (14) 


$$
\mathbf{J}=\left[\begin{array}{cccc}
0 & 0 & 0 & 1 \\
R-(R-1) x_{3}^{*} & -1 & -(R-1) x_{1}^{*} & 0 \\
4 \gamma x_{2}^{*} & 4 \gamma x_{1}^{*} & -4 \gamma & 0 \\
\sigma\left[\left(R-\xi^{-1}\right)-(R-1) x_{3}^{*}\right] & \sigma\left(\xi^{-1}-1\right) & -\sigma(R-1) x_{1}^{*} & \sigma \delta+\xi^{-1}
\end{array}\right] .
$$

evaluated at equilibrium points are :

$$
\left\{\begin{aligned}
E_{1}=(0 ; 0 ; 0 ; 0): & \lambda_{1}=-1, \quad \lambda_{2}=-38.5943, \\
& \lambda_{3}=59.5943, \quad \lambda_{4}=2 . \\
E_{2}=(1 ; 1 ; 1 ; 0): & \lambda_{1}=-2.6376, \\
& \lambda_{2}=-10.0396 \\
\lambda_{3,4}=15.3386 \pm 10.59 j . & \\
E_{3}=(-1 ;-1 ; 1 ; 0): & \text { has the same eigenvalues as } \\
& \text { the equilibrium } E_{2} .
\end{aligned}\right.
$$

The eigenvalues $\lambda_{1}, \lambda_{2}, \lambda_{3}$ and $\lambda_{4}$ show that the equilibrium $E_{1}$ is a saddle point, the equilibria $E_{2}$ and $E_{3}$ are saddle-focus points. According to the stability condition (9), where $\alpha_{1}=\alpha_{2}=\alpha_{3}=\alpha_{4}=1$, we have eigenvalues for equilibria $E_{1}, E_{2}$ and $E_{3}$ in the unstable region and therefore we can confirm the chaotic behavior of the integer-order system (13) of the thermal convection in a viscoelastic fluidsaturated porous medium. In Fig. 1 are depicted the simulation results of the (integer-order) system (13) with the initial conditions $\left(x_{1}(0), x_{2}(0), x_{3}(0), x_{3}(0)\right)=[0.9,0.9,0.9,0.1]$.
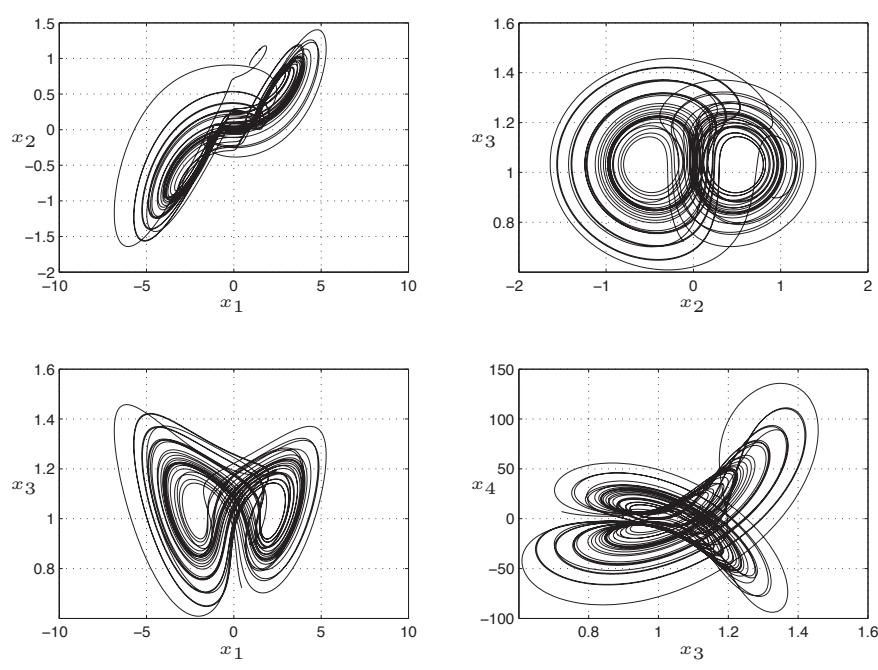

Fig. 1. Simulation results of the (integer-order) system (13) in $x_{1}-x_{2}$, $x_{2}-x_{3}, x_{1}-x_{3}$ and $x_{3}-x_{4}$ planes for parameters given in (15).

\section{B. Fractional-order system of the thermal convection in a viscoelastic fluid-saturated porous medium}

Based on model (13), we propose in this section a new fractional viscoelastic fluids model in porous media. The total order of the system has changed from 4 to the sum of each particular order. To put this fact into context, we propose the fractional-order dynamical model of the thermal convection in a viscoelastic fluid-saturated porous medium with zero initial value history.

$$
\left\{\begin{aligned}
{ }_{0} D_{t}^{\alpha_{1}} x_{1} & =x_{4}, \\
{ }_{0} D_{t}^{\alpha_{2}} x_{2} & =R x_{1}-x_{2}-(R-1) x_{1} x_{3}, \\
{ }_{0} D_{t}^{\alpha_{3}} x_{3} & =4 \gamma\left(x_{1} x_{2}-x_{3}\right), \\
{ }_{0} D_{t}^{\alpha_{4}} x_{4} & =\sigma\left[\left(R-\xi^{-1}\right) x_{1}+\left(\xi^{-1}-1\right) x_{2}\right. \\
\left.\quad-(R-1) x_{1} x_{3}-\left(\delta+\frac{1}{\sigma \xi}\right) x_{4}\right], &
\end{aligned}\right.
$$

where $\alpha_{1}, \alpha_{2}, \alpha_{3}$ and $\alpha_{4}$ are the derivatives orders. The total order of the system is $\bar{\alpha}=\left(\alpha_{1}, \alpha_{2}, \alpha_{3}, \alpha_{4}\right)$.

Numerical solution of the fractional-order dynamical model of the thermal convection in a viscoelastic fluidsaturated porous system (17) is given by (18), where $T_{s}$ is the time simulation by setting $N=\left[T_{s} / h\right], k=1,2, \cdots, N$ and $\left(x_{1}(0), x_{2}(0), x_{3}(0), x_{4}(0)\right)$ are the initial conditions. The binomial coefficients $c_{j}^{\left(\alpha_{i}\right)}, \forall i$ are calculated according to relation (3).

1) Commensurate order system: Let us consider the commensurate order system (17) with $\alpha_{1}=\alpha_{2}=\alpha_{3}=\alpha_{4}=0.9$ and the system parameters given in (15).

The stability of all equilibrium points should be investigated. Because of the system parameters given in (15), the equilibrium points are the same as in the case of integerorder system. The stability can be investigated according to condition (9). Hence the equilibria $E_{2}$ and $E_{3}$ are saddlefocus points. According to the stability condition (9), where $\alpha_{1}=\alpha_{2}=\alpha_{3}=\alpha_{4}=0.9$, we have eigenvalues $\mid \arg \left(\lambda_{1} \mid<\right.$ $\frac{\alpha \pi}{2}, \mid \arg \left(\lambda_{2} \mid<\frac{\alpha \pi}{2}\right.$ and $\mid \arg \left(\lambda_{3,4} \mid<\frac{\alpha \pi}{2}\right.$ for the equilibrium $E_{2}$ and eigenvalues $\mid \arg \left(\lambda_{1}\left|<\frac{\alpha \pi}{2},\right| \arg \left(\lambda_{2} \mid<\frac{\alpha \pi}{2}\right.\right.$ and $\mid \arg \left(\lambda_{3,4} \mid<\frac{\alpha \pi}{2}\right.$ for the equilibrium $E_{3}$ in unstable region and therefore we can confirm the chaotic behavior of system (17).

As we can see, the condition to have at least one root in the unstable region in order for the system to be chaotic is satisfied for the two equilibria points $E_{2}$ and $E_{3}$. In Fig. 2 is depicted a chaotic attractor of the new fractionalorder system (17) for the system parameters given in (15), derivative orders $\alpha_{1}=\alpha_{2}=\alpha_{3}=\alpha_{4}=0.9$ for time step $h=0.0005$ and iteration number $N=10000$.

The total order $\bar{\alpha}=3.6$ of the fractional-order system is the sum of the orders of all involved derivatives. When $\alpha_{i}$ $(i=1,2,3,4)$, the system (17) is the original integer-order system, which is chaotic with the parameters given in (15). We can see that the chaotic attractor in the new fractionalorder system (17) is similar and looks like the corresponding integer-order system.

2) Non-commensurate order system: When we assume the different orders of derivatives in the fractional-order 


$$
\left\{\begin{array}{l}
x_{1}\left(t_{k}\right)=\left(x_{4}\left(t_{k-1}\right) h^{\alpha_{1}}-\sum_{j=1}^{k} c_{j}^{\left(\alpha_{1}\right)} x_{1}\left(t_{k}-j\right),\right. \\
x_{2}\left(t_{k}\right)=\left(R x_{1}\left(t_{k}\right)-x_{2}\left(t_{k-1}\right)-(R-1) x_{1}\left(t_{k}\right) x_{3}\left(t_{k-1}\right)\right) h^{\alpha_{2}}-\sum_{j=1}^{k} c_{j}^{\left(\alpha_{2}\right)} x_{2}\left(t_{k}-j\right), \\
x_{3}\left(t_{k}\right)=\left[4 \gamma\left(x_{1}\left(t_{k}\right) x_{2}\left(t_{k}\right)-x_{3}\left(t_{k-1}\right)\right)\right] h^{\alpha_{3}}-\sum_{j=1}^{k} c_{j}^{\left(\alpha_{3}\right)} x_{3}\left(t_{k}-j\right), \\
x_{4}\left(t_{k}\right)=\left(\sigma\left[\left(R-\xi^{-1}\right) x_{1}\left(t_{k}\right)+\left(\xi^{-1}-1\right) x_{2}\left(t_{k}\right)-(R-1) x_{1}\left(t_{k}\right) x_{3}\left(t_{k}\right)-\left(\delta+\frac{1}{\sigma \xi}\right) x_{4}\left(t_{k-1}\right)\right]\right) h^{\alpha_{4}}-\sum_{j=1}^{k} c_{j}^{\left(\alpha_{4}\right)} x_{4}\left(t_{k}-j\right) .
\end{array}\right.
$$
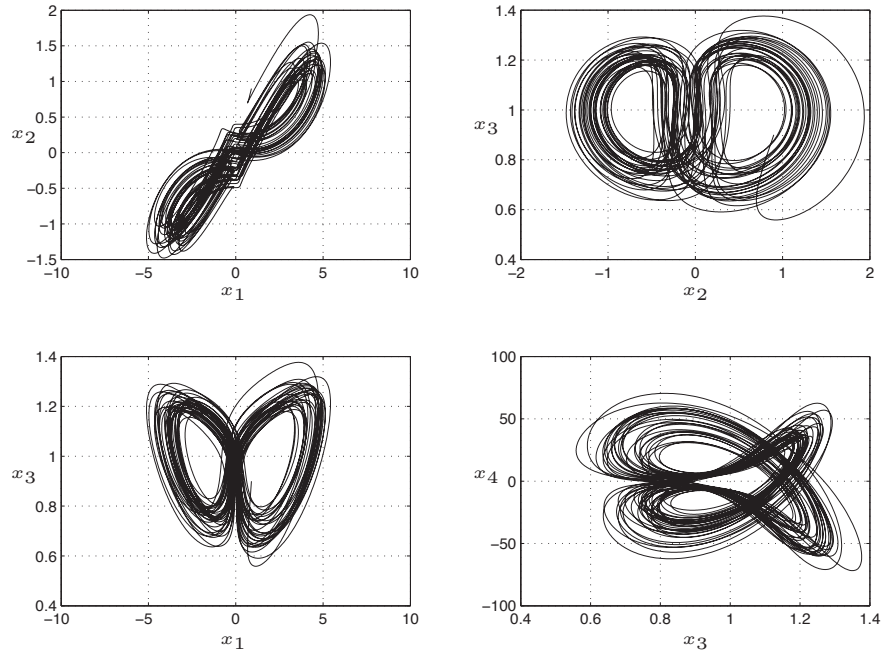

Fig. 2. Simulation results of the fractional-order system (17) in $x_{1}-x_{2}$, $x_{2}-x_{3}, x_{1}-x_{3}$ and $x_{3}-x_{4}$ planes for orders $\alpha_{1}=\alpha_{2}=\alpha_{3}=\alpha_{4}=0.9$ and parameters given in (15).

system (17), i.e. $\alpha_{1} \neq \alpha_{2} \neq \cdots \neq \alpha_{n}$, we get a general non-commensurate order system. There is no exact condition for determining the orders to obtain chaotic behavior of the system. The equilibrium points are the same as in the case of integer-order system with the system parameters given in (15). The stability can be investigated according to characteristic equation (11). Now, let us consider the noncommensurate order system (17) with $\alpha_{1}=0.97, \alpha_{2}=0.89$, $\alpha_{3}=0.98, \alpha_{4}=0.85$ and the parameters given in (15).

For above derivative orders and the system parameters, and for the Jacobian matrix $\mathbf{J}$ (14) evaluated at the equilibrium points (16), equation (11) becomes as follows

$$
\operatorname{det}\left(\operatorname{diag}\left(\left[\begin{array}{llll}
\lambda^{97} & \lambda^{89} & \lambda^{98} & \lambda^{85}
\end{array}\right]\right)-\mathbf{J}\right)=0 .
$$

The characteristic equation of system (17) evaluated at the equilibrium $E_{2}$ and $E_{3}$ is

$$
\begin{aligned}
\lambda^{369}- & 21 \lambda^{284}+\lambda^{280}+2 \lambda^{271}-21 \lambda^{195}-42 \lambda^{186} \\
+ & 48 \lambda^{182}-1008 \lambda^{97}+4600 \lambda^{89}+9200=0 .
\end{aligned}
$$

The above characteristic equation is polynomial of very high order and it is difficult to find the roots of such polynomial. For the system to remain chaotic, there should be at least one root $\lambda$ in the unstable region, which means that $|\arg (\lambda)|<$ $\frac{\pi}{2 m}$ where $m=100$ (LCM of orders denominator). Because of roots calculation problem, we can predict one unstable eigenvalue and assume that the stability condition for chaos is satisfied. It can be indirectly proved via the chaotic attractor, which can be observed in Fig. 3 with the system parameters given in (15), non-commensurate orders of the derivatives being : $\alpha_{1}=0.97, \alpha_{2}=0.89, \alpha_{3}=0.98$ and $\alpha_{4}=0.85$ for time step $h=0.0005$ and iteration number $N=10000$. The total order of the system is $\bar{\alpha}=3.69$.
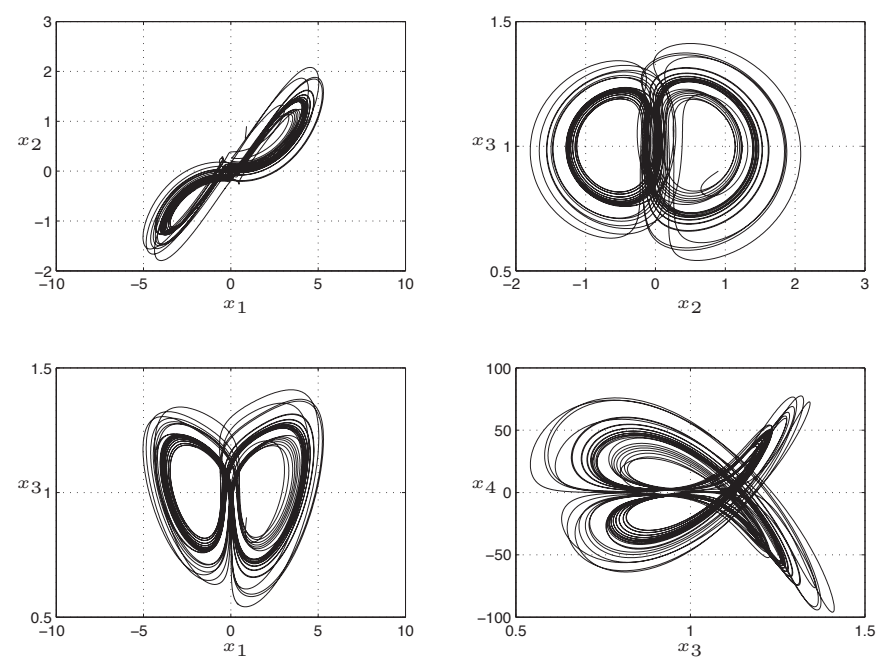

Fig. 3. Simulation results of the fractional-order system (17) in $x_{1}-x_{2}$, $x_{2}-x_{3}, x_{1}-x_{3}$ and $x_{3}-x_{4}$ planes for orders $\alpha_{1}=0.97, \alpha_{2}=0.89$, $\alpha_{3}=0.98, \alpha_{4}=0.85$ and parameters given in (15).

\section{Bifurcation and chaos versus the Darcy-Rayleigh num- ber $R$}

The dynamical behaviors of the fractional-order system (17) are investigated numerically by means of bifurcation diagrams. Fig. 4 shows the $x_{1}-x_{3}$ projection for different values of the modified Darcy-Rayleigh number $R$ for orders $\alpha_{1}=0.97, \alpha_{2}=0.89, \alpha_{3}=0.98$ and $\alpha_{4}=0.85$. Fig. 4 illustrates the transition from steady convection to chaos. It can be seen from Fig. 4a that for $R=4$ the solution trajectory spiral toward the fixed point, resulting in steady convection. Fig. 4b inset of Fig. 4a detailing the oscillatory decay of the solution for $R=9$. Moreover, when $R=9.1$ a transition from a periodic solution to chaos is obtained which prevails over a wide range of values of $R$ as illustrated in Figs. 4c and 4d. The post transient solution trajectory presented in Fig. 4e with $R=50$ exhibits typical chaotic behavior, showing that the path depicted does not approach 
a periodic limit or an equilibrium point. However, when $R=200$ the chaos have been intensified as illustrated in Fig. 4f. The bifurcation diagrams are shown in Fig. 5. As we can see in Fig. 5, the value of the modified Darcy-Rayleigh number $R$ affects the system behavior, when the DarcyRayleigh number $R$ is small the system exhibits steady state response, the first bifurcation from chaos beginning at $R \approx 24$ and as $R$ increases, the system exhibits chaotic behavior.
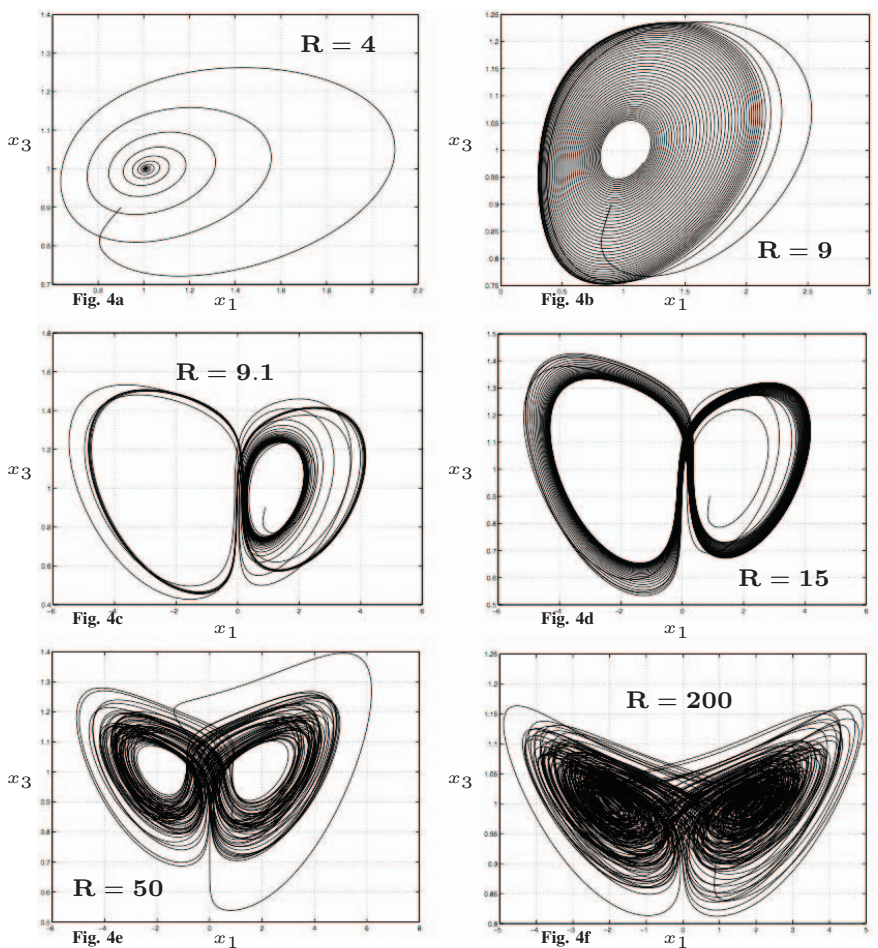

Fig. 4. Simulation results of the fractional-order system (17) in $x_{1}-x_{3}$ plane for orders $\alpha_{1}=0.97, \alpha_{2}=0.89, \alpha_{3}=0.98, \alpha_{4}=0.85$ and for different values of $R$.

From Fig. 6, the Fourier power spectrum diagrams of the fractional-order system (17) show spectral broadening. It can be seen that the presence of pronounced spikes in the diagrams indicates periodic motion which is to be chaotic.

\section{CONCLUSION}

In this paper we have proposed a new fractional-order dynamical model of the thermal convection in a viscoelastic fluid-saturated porous medium, clearly leading to chaotic behavior. The new generalized fractional-order system can be reduced to an equivalent fractional-order Khayat's system, which describes the thermal convection in a pure viscoelastic fluid layer, with the only differences being in parameter interpretation. This can be reduced to many systems, the fractional-order Vadasz's system and the fractional-order Akhatov's system. The behavior and stability analysis of the integer-order and the fractional commensurate and non-
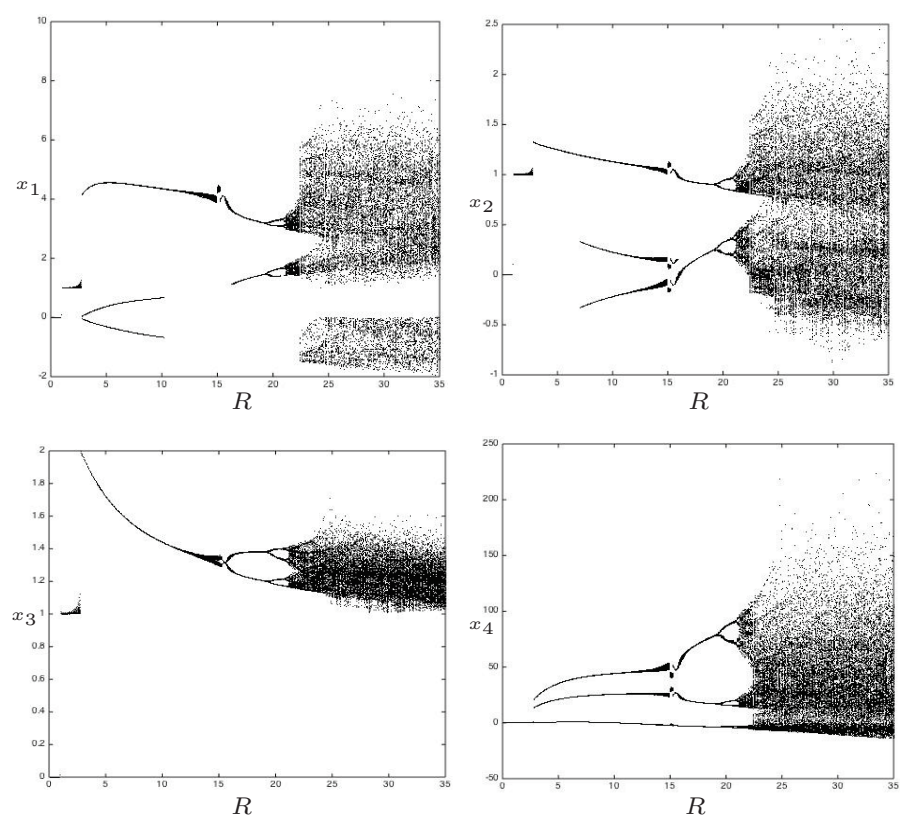

Fig. 5. Bifurcation diagram of the fractional-order system (17) for orders $\alpha_{1}=0.97, \alpha_{2}=0.89, \alpha_{3}=0.98, \alpha_{4}=0.85$.
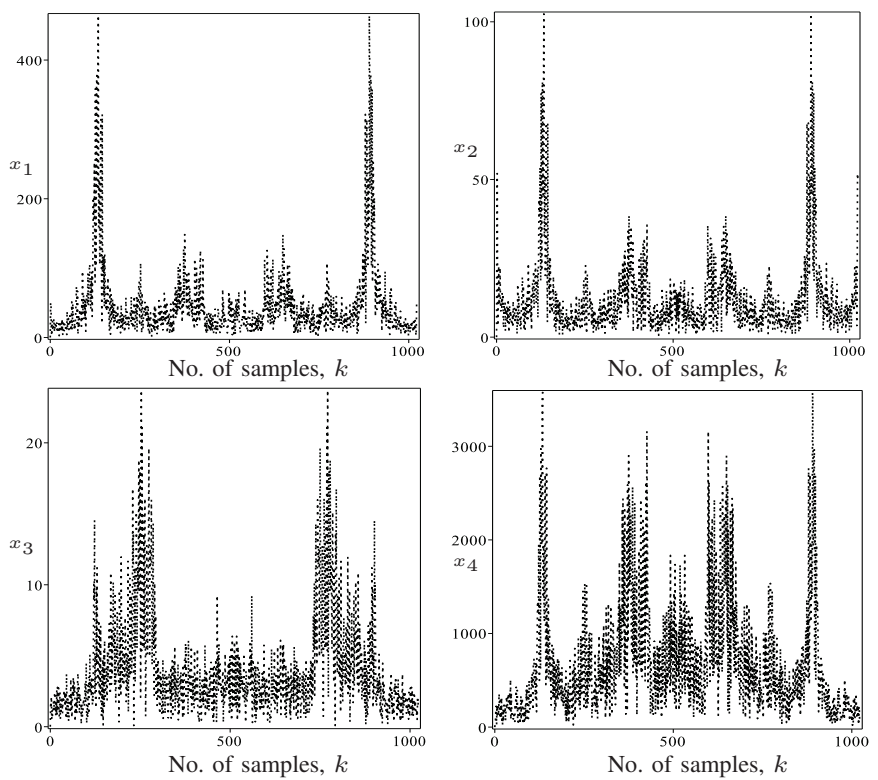

Fig. 6. Fourier power spectrum diagrams of the fractional-order system (17) for orders $\alpha_{1}=0.97, \alpha_{2}=0.89, \alpha_{3}=0.98, \alpha_{4}=0.85$ and parameters given in (15).

commensurate order system with total order less than 4 , which exhibits chaos, have been presented.

\section{ACKNOWLEDGEMENT}

The research reported herein was supported by the King Abdullah University of Science and Technology (KAUST).

\section{REFERENCES}

[1] L. J. Sheu, L. M. Tam, J. H. Chen, H. K. Chen, K. T. Lin, and Y. Kang, "Chaotic convection of viscoelastic fluids in porous media," Chaos, Solitons \& Fractals, vol. 37, pp. 113-124, 2008. 
[2] M. S. Malashetty, I. S. Shivakumara, S. Kulkarni, and M. Swamy, "Convective instability of oldroyd-B fluid saturated porous layer heated from below using a thermal non-equilibrium model," Transport in Porous Media, vol. 64, pp. 123-139, 2006.

[3] E. N. Lorenz, "Deterministic non periodic flow," Journal of the Atmospheric Sciences, vol. 20, pp. 130-141, 1963.

[4] J. Stavans, F. Heslot, and A. Libchaber, "Fixed winding number and the quasi periodic route to chaos in a convective fluid," Physical Review Letters, vol. 55, pp. 596-599, 1985.

[5] A. E. Deane, E. Knobloch, and J. Toomre, "Travelling waves and chaos in thermosolutal convection," Physical Review A, vol. 36, pp. 28622869, 1987.

[6] L. J. Sheu, "An autonomous system for chaotic convection in a porous medium using a thermal non-equilibrium model," Chaos, Solitons \& Fractals, vol. 30, pp. 672-689, 2006.

[7] D. Laroze, P. Siddheshwar, and H. Pleiner, "Chaotic convection in a ferrofluid," Commun Nonlinear Sci. Numer. Simulat., vol. 18, pp. 2436-2447, 2013.

[8] P. G. Siddheshwar and D. Radhakrishna, "Linear and nonlinear electroconvection under ac electric field," Commun Nonlinear Sci. Numer. Simulat., vol. 17, pp. 2883-2895, 2012.

[9] P. Vadasz, "Local and global solutions for transitions to chaos and hysteresis in a porous layer heated from below," Transport in Porous Media, vol. 37, pp. 213-245, 1999.

[10] P. Vadasz and S. Olek, "Weak turbulence and chaos for low Prandtl number gravity driven convection in porous media," Transport in Porous Media, vol. 37, pp. 69-91, 1999.

[11] P. Vadasz and S. Olek, "Route to chaos for moderate Prandtl number convection in a porous layer heated form below," Transport in Porous Media, vol. 41, pp. 211-239, 2000.

[12] R. C. Koeller, "Applications of fractional calculus to the theory of viscoelasticity," Journal of Applied Mechanics, vol. 51, pp. 299-307, 1984.

[13] D. Y. Song and T. Q. Jiang, "Study on the constitutive equation with fractional derivative for the viscoelastic fluids - modified Jaffrays model and its application," Rheologica Acta, vol. 37, pp. 512-517, 1998.

[14] W. H. Park, J. Choe, and J. M. Kang, "Pressure behavior of transport in fractal porous media using fractional calculus approach," Energy Sources, vol. 22, pp. 881-890, 2000.

[15] Q. Wang and D. Tong, "The flow analysis of viscoelastic fluid with fractional order derivative in horizontal well," Transport in Porous Media, vol. 81, pp. 295-303, 2010.

[16] D. Tripathi and O. A. Beg, "A numerical study of oscillating peristaltic flow of generalized Maxwell viscoelastic fluids through a porous medium," Transport in Porous Media, vol. 95, pp. 337-348, 2012.

[17] Y. Liu, L. Zheng, and X. Zhang, "Unsteady MHD Couette flow of a generalized Oldroyd-B fluid with fractional derivative," Computers \& Mathematics with Applications, vol. 61, pp. 443-450, 2011.

[18] C. Li and G. Chen, "Chaos in the fractional order Chen system and its control," Chaos, Solitons \& Fractals, vol. 22, pp. 549-554, 2004.

[19] I. Petrás̆, "A note on the fractional-order Chua's system," Chaos, Solitons \& Fractals, vol. 38, pp. 140-147, 2008.

[20] T. Hartley, C. Lorenzo, and H. Qammer, "Chaos in a fractional order Chua's system," IEEE Trans. Circ. Syst. I : Fund. Theory \& Appli., vol. 42, pp. 485-490, 1995.

[21] P. Arena, R. Caponetto, L. Fortuna, and D. Porto, "Bifurcation and chaos in non-integer order cellular neural networks," Int. J. Bifurcation \& Chaos, vol. 8, pp. 1527-1539, 1998.

[22] W. M. Ahmad and R. El-Khazali, "Chaos in the fractional order periodically forced complex duffing's oscillators," Chaos, Solitons \& Fractals, vol. 24, pp. 1097-2104, 2005.

[23] I. Petrás̆, "A note on the fractional-order Volta system," Commun Nonlinear Sci. Numer. Simulat., vol. 15, pp. 384-393, 2010.

[24] W. M. Ahmad, "Hyperchaos in fractional order nonlinear systems," Chaos, Solitons \& Fractals, vol. 26, pp. 1459-1465, 2005.

[25] R. S. Barbosa, J. A. Tenreiro Machado, B. M. Vinagre, and A. J. Calderon, "Hyperchaos in fractional order nonlinear systems," Journal of Vibration and Control, vol. 26, pp. 1459-1465, 2007.

[26] C. Li and J. Yan, "The synchronization of three fractional differential systems," Chaos, Solitons \& Fractals, vol. 32, pp. 751-757, 2007.

[27] I. Podlubny, Fractional Differential Equations. New York: Academic Press, 1999.

[28] I. Petrás̆, Fractional-Order Nonlinear Systems : Modeling, Analysis and Simulation. Berlin: Springer, 2011.
[29] W. Deng, "Short memory principle and a predictor-corrector approach for fractional differential equations," J. Comput. Appl. Math., vol. 206, pp. 174-188, 2007.

[30] L. Dorckák, "Numerical models for simulation of the fractional-order control systems," Tech. Rep. UEF-04-94, Institute of Experimental Physics, Academy of Sciences, Slovakia, 1994.

[31] S. M. Tavazoei and M. Haeri, "A necessary condition for double scroll attractor existence in fractional-order systems," Physics Letters A, vol. 367, pp. 102-113, 2007.

[32] S. M. Tavazoei and M. Haeri, "Limitations of frequency domain approximation for detecting chaos in fractional order systems," Nonlinear Analysis, vol. 69, pp. 1299-1320, 2008.

[33] Y. Li, Y. Chen, and I. Podlubny, "Stability of fractional-order nonlinear dynamic systems : Lyapunov direct method and generalized Mittag-Leffler stability," Computers \& Mathematics with Applications, vol. 59, pp. 1810-1821, 2010. 